\title{
The Method of Exterior Forms in Linear Programming
}

\author{
Gennadii V. Kondratiev ${ }^{1, a}$ \\ ${ }^{1} 24$ Minina St., State Technical University of Nizhniy Novgorod after R.E. Alexeev, Russia \\ agennadii.kondratiev@gmail.com
}

Keywords: linear dependency, exterior form, linear programming, special canonical form.

\begin{abstract}
The method of exterior forms by H. Grassmann and E. Cartan is used for solving the linear programming problem. It captures the essence of the problem in a convenient, compact form. The solution is presented by Cramer's like rules and is reduced to computing the set of values of the objective function at the vertices of the polyhedron constraints, without any explicit calculation of the vertices themselves.
\end{abstract}

\section{Introduction}

The linear programming problem represents a rare example of completely decidable problem in which either existence or absence of the solution is being established in a finite number of steps. This is due to the fact that the number of points that are suspicious for the extremum is finite and effectively definable. It is well known that these points are the vertices of a convex polyhedron on which the linear function is being searched for the extrema $[1,2,3]$.

A direct search of all vertices is a valid procedure, which gives the detailed information about all the corner points and the value of the function being optimized. However, in large-scale problems with a sufficiently large number of constraints the quantity of corner points can be overwhelming. The famous G. B. Danzig simplex method [1] has the problem reduced to the turn-based optimization, strictly approximating the value of the function to the extreme, and therefore does not requiring verification of all vertices of the polyhedron.

The proposed method of exterior forms due to genious of H. Grassmann and E. Cartan [4,5] reduces geometry to multilinear algebra and allows to write explicit formulas, similar to Cramer's rules for the solution of linear equations, to calculate the values of the objective function at the vertices of the polyhedron through determinants. Although, it does not much decreases the amount of calculations in comparison with the full search (approximately, just by one order), the simplicity, clearness, and good programmability of the algorithm, as well as the ability to see the values of the objective function at all corner points can be an advantage in applications. Together with the value of the objective function, the set of free variables for each vertex is automatically determined. The algorithm stops with the choice of the extreme value of the objective function, being valid, that is such a value where all the coordinates of the corner point are nonnegative. It is assumed that the problem is presented in a special canonical form.

\section{Linear Dependency and Exterior Product}

In this section we recall the properties of the exterior product of vectors [4,5].

Definition 1. Let $V$ be a real $n$-dimensional linear space. The exterior algebra $\Lambda(V)$ over the space $V$ is a free graded $R$-algebra with multiplication $\wedge: \Lambda(V) \times \Lambda(V) \rightarrow \Lambda(V)$, such that:

- $\Lambda(V)$ is a direct sum of linear spaces $\bigoplus_{i=0}^{n} \Lambda^{i}(V)$, where $\Lambda^{0}(V)=\mathbb{R}, \Lambda^{1}(V)=V$,

- $\Lambda^{i}(V)=V \wedge V \wedge \ldots \wedge V$ ( $i$ times), $1<i \leq n,-$ the $i$-th exterior power of the space $V$, that is a linear space generated by all the products $\alpha_{1}^{1} \wedge \ldots \wedge \alpha_{i}^{1}$, where $\alpha_{k}^{1} \in V, 1 \leq k \leq i$, subject to constraints of associativity and bilinearity of the product $\wedge$, 
- for any two subspaces $\Lambda^{i}(V), \quad \Lambda^{j}(V), \quad 0 \leq i, j \leq n$, the value of the product $\wedge: \Lambda^{i}(V) \times \Lambda^{j}(V) \rightarrow \Lambda(V):\left(\alpha^{i}, \alpha^{j}\right) \mapsto \alpha^{i} \wedge \alpha^{j}$ belongs to the subspace $\Lambda^{i+j}(V)$,

- the multiplication $\wedge$ is anticommutative, that is for any two elements $\alpha^{i} \in \Lambda^{i}(V), \alpha^{j} \in \Lambda^{j}(V)$ the following holds $\alpha^{i} \wedge \alpha^{j}=(-1)^{i \cdot j} \alpha^{j} \wedge \alpha^{i}$.

Multiplication $\wedge$ is called exterior. The dimension of the space $\Lambda^{i}(V), 0 \leq i \leq n$, is equal to the number of the basis vectors $e_{j_{1}} \wedge e_{j_{2}} \wedge \cdots \wedge e_{j_{i}}, 1 \leq j_{1}<j_{2}<\cdots<j_{i} \leq n$, where $\left\{e_{1}, \ldots, e_{n}\right\}--$ the basis of the space $V$, that is $\operatorname{dim}\left(\Lambda^{i}(V)\right)=C_{n}^{i}=\frac{n !}{i !(n-i) !}$. Accordingly, the dimension of the exterior algebra $\Lambda(V)$ is $2^{n}$.

Exterior multiplication allows to express the fact of linear dependency of the vectors in the form of equality.

Lemma 1. Let $\left\{\alpha_{1}, \ldots, \alpha_{m}\right\}$ be a finite non-empty set of linearly independent vectors of the space $V, \operatorname{dim}(V)=n, m \leq n$. A vector $\beta \in V$ is linearly dependent on the vectors $\alpha_{1}, \ldots, \alpha_{m}$ if and only if $\beta \wedge \alpha_{1} \wedge \cdots \wedge \alpha_{m}=0$.

Proof. If $\beta=\sum_{i=1}^{m} c_{i} \alpha_{i}$, then $\beta \wedge \alpha_{1} \wedge \cdots \wedge \alpha_{m}=\sum_{i=0}^{m} c_{i} \alpha_{i} \wedge \alpha_{1} \wedge \cdots \wedge \alpha_{m}=0$.

Conversely, suppose that $\beta \wedge \alpha_{1} \wedge \cdots \wedge \alpha_{m}=0$. Add to the set $\left\{\alpha_{1}, \ldots, \alpha_{m}\right\}$ new vectors $\alpha_{m+1}, \ldots, \alpha_{n}$ to complete it to a basis of the vector space $V$. Then $\beta=\sum_{i=1}^{n} c_{i} \alpha_{i}$, and the equation $\sum_{i=m+1}^{n} c_{i} \alpha_{i} \wedge \alpha_{1} \wedge \cdots \wedge \alpha_{m}=0$ implies $c_{m+1}, \ldots, c_{n}=0$.

Proposition 1. Suppose that $\left\{e_{1}, \ldots, e_{n}\right\}$ is a basis of the linear space $V$. The exterior product of the vectors $\alpha_{i}=a_{i}^{j} e_{j}, i=1, \ldots, m, m \leq n$, is equal to zero $\alpha_{1} \wedge \cdots \wedge \alpha_{m}=0$ if and only if all the $m \times m$-determinants $\Delta_{k_{1} \ldots k_{m}}$ of the matrix $\left(\begin{array}{ccc}a_{1}^{1} & \cdots & a_{m}^{1} \\ a_{1}^{2} & \cdots & a_{m}^{2} \\ & \cdots & \\ a_{1}^{n} & \cdots & a_{m}^{n}\end{array}\right)$ are equal to zero. The determinant $\Delta_{k_{1} \ldots k_{m}}$ is made of the rows with numbers $k_{1}, \ldots, k_{m}$.

The proof follows from the expansion of the exterior product of the vectors through the basis ones. The coefficients of this expansion are the required determinants.

The number of the determinants $\Delta_{k_{1} \ldots k_{m}}$ is given by $C_{n}^{m}=\frac{n !}{m !(n-m) !}$.

\section{The Linear Programming Problem}

The linear programming problem in the standard form is formulated in the following way $[1,2,3]$. It is required to find an extreme (minimum or maximum), if it exists, of a linear function

$$
J(x)=\sum_{i=1}^{n} c_{i} x^{i} \rightarrow \text { ext }
$$

subject to constraints

$$
\begin{aligned}
& \sum_{i=1}^{n} a_{i}^{j} x^{i}=b^{j}, j=1, \ldots, m \\
& x^{i} \geq 0, i=1, \ldots, n
\end{aligned}
$$

where $m<n$ and equations (2) are linearly independent. 
Further, a simple case, when the linear variety determined by equations (2) passes through the origin, is eliminated from the consideration. In that case, there is only one corner point of the system of equations and inequalities (2) - (3), the origin, which is easy to be checked on the extreme.

Since not all $b^{j}, j=1, \ldots, m$, are zero, equations (2) can be rewritten in the equivalent form

$$
\begin{aligned}
& \sum_{i=0}^{n} d_{i}^{1} x^{i}=1 \\
& \sum_{i=0}^{n} d_{i}^{j} x^{i}=0, \quad j=2, \ldots, m
\end{aligned}
$$

It is known that the function $J(x)$ reaches an extremum, when it exists, at one or more corner points. The corner points of the system (2) - (3) or ( $\left.2^{\prime}\right)$ - (3) are determined by equations (2) or ( $\left.2^{\prime}\right)$, supplemented by the vanishing of $n-m$ variables $x^{k_{1}}=0, x^{k_{2}}=0, \ldots, x^{k_{n-m}}=0$, $1 \leq k_{1}<k_{2}<\cdots<k_{n-m} \leq n$.

Proposition 2. The set of values of the objective function (1) at the corner points of set $\left(2^{\prime}\right)-(3)$

belongs to a finite set of fractions $\left\{\frac{\Delta_{k_{1} \cdots k_{m}}^{1}}{\Delta_{k_{1} \cdots k_{m}}^{2}} \mid 1 \leq k_{1}<k_{2}<\cdots<k_{m} \leq n\right\}$, where $\Delta_{k_{1} \ldots k_{m}}^{1}, \Delta_{k_{1} \ldots k_{m}}^{2}$ are the determinants composed of the columns with numbers $k_{1}, k_{2}, \ldots, k_{m}$ of the matrices $\left(\begin{array}{ccc}c_{1} & \ldots & c_{n} \\ d_{1}^{2} & \ldots & d_{n}^{2} \\ & \ldots & \\ d_{1}^{m} & \ldots & d_{n}^{m}\end{array}\right)$ and $\left(\begin{array}{ccc}d_{1}^{1} & \ldots & d_{n}^{1} \\ d_{1}^{2} & \ldots & d_{n}^{2} \\ & \ldots & \\ d_{1}^{m} & \ldots & d_{n}^{m}\end{array}\right)$, respectively.

Remark. If the fraction $\frac{\Delta_{k_{1} \ldots k_{m}}^{1}}{\Delta_{k_{1} \ldots k_{m}}^{2}}$ is not defined, this element is regarded as a symbol. The elements of the form $\frac{r}{0}, \frac{-r}{0}, r \in \mathbb{R}, r>0$, indicate the absence of a maximum or minimum of the objective function, respectively.

Proof. Introduce the notation $v=x^{1} e_{1}+x^{2} e_{2}+\cdots+x^{n} e_{n}, \quad \gamma=c_{1} e^{1}+c_{2} e^{2}+\cdots+c_{n} e^{n}$, $\delta^{j}=d_{1}^{j} e^{1}+d_{2}^{j} e^{2}+\cdots+d_{n}^{j} e^{n}, j=1, \ldots, m$, where $\left\{e_{1}, \ldots, e_{n}\right\}$ and $\left\{e^{1}, \ldots, e^{n}\right\}$ are the dual bases of the linear space $V$ and its dual $V^{*}$. Let $\langle\cdot \mid \cdot\rangle: V^{*} \times V \rightarrow \mathbb{R}:(\alpha, w) \mapsto\langle\alpha \mid w\rangle$ be the bilinear pairing of covectors and vectors. Then, at the corner point of the system $\left(2^{\prime}\right)-(3)$, defined by the equations $x^{k_{1}}=0, \ldots, x^{k_{n-m}}=0$, the following relations hold

$$
\begin{gathered}
\left\langle\delta^{1} \mid v\right\rangle=1, \\
\left\langle\delta^{j} \mid v\right\rangle=0, j=2, \ldots, m, \\
\left\langle e^{k_{l}} \mid v\right\rangle=0, l=1, \ldots, n-m .
\end{gathered}
$$

The kernel $\operatorname{ker}(\langle\cdot \mid v\rangle)$ of the linear form $\langle\cdot \mid v\rangle: V^{*} \rightarrow \mathbb{R}$ is a linear subspace of dimension $n-1$ of the space $V^{*}$, generated by the covectors $\delta^{j}, e^{k_{l}}, j=2, \ldots, m, l=1, \ldots, n-m$. For any covector $v^{*} \in V^{*}$ there exists a number $\lambda \in \mathbb{R}$, such that the equality $v^{*}=\lambda \cdot \delta^{1} \bmod (\operatorname{ker}(\langle\cdot \mid v\rangle))$ holds, that is $v^{*}-\lambda \cdot \delta^{1} \in \operatorname{ker}(\langle\cdot \mid v\rangle)$. Consequently, $\quad\left(v^{*}-\lambda \cdot \delta^{1}\right) \wedge \delta^{2} \wedge \ldots \wedge \delta^{m} \wedge e^{k_{1}} \wedge \ldots \wedge e^{k_{n-m}}=0, \quad$ where $\lambda=\left\langle v^{*} \mid v\right\rangle$. The $n$-form at the left-hand side of the equality is a product of the volume form with a 
scalar, that is an expression of the form $\lambda_{k_{1}^{\prime} \ldots k_{m}^{\prime}} \cdot e^{1} \wedge \cdots \wedge e^{n}$, where $\left\{k_{1}^{\prime}, \ldots, k_{m}^{\prime}\right\}$ is the set of indices, complementary to the set $\left\{k_{1}, \ldots, k_{n-m}\right\}$ in the set $\{1, \ldots, n\}$.

The number $\lambda_{k_{1}^{\prime} \ldots k_{m}^{\prime}}$ coincides with the coefficient in the expansion of the $m$-form $\left(v^{*}-\lambda \cdot \delta^{1}\right) \wedge \delta^{2} \wedge \ldots \wedge \delta^{m}=\sum_{1 \leq k_{1}^{\prime}<\ldots<k_{m}^{\prime} \leq n} \lambda_{k_{1}^{\prime} \ldots k_{m}^{\prime}} \cdot e^{k_{1}^{\prime}} \wedge \cdots \wedge e^{k_{m}^{\prime}}$

Suppose $v^{*}=\gamma$, then $\lambda_{k_{1}^{\prime} \ldots k_{m}^{\prime}}=\Delta_{k_{1}^{\prime} \ldots k_{m}^{\prime}}^{1}-\lambda \cdot \Delta_{k_{1}^{\prime} \ldots k_{m}^{\prime}}^{2}$, where $1 \leq k_{1}^{\prime}<\ldots<k_{m}^{\prime} \leq n$. The vanishing of the coefficient $\lambda_{k_{1}^{\prime} \ldots k_{m}^{\prime}}=0$ determines the linear equation with respect to the variable $\lambda=\langle\gamma \mid v\rangle$, where $v$ is the vector satisfying equation $(*)$. Consequently, the set of values of the objective function (1) at the corner points of the domain of definition (2') - (3) belongs to the set $\left\{\frac{\Delta_{k_{1} \ldots k_{m}}^{1}}{\Delta_{k_{1} \ldots k_{m}}^{2}} \mid 1 \leq k_{1}<k_{2}<\cdots<k_{m} \leq n\right\} . C_{n}^{m}=\frac{n !}{m !(n-m) !}$ is the number of elements in the latter set.

The value of the objective function, taken from the set $\left.\frac{\Delta_{k_{1} \ldots k_{m}}^{1}}{\Delta_{k_{1} \ldots k_{m}}^{2}} \mid 1 \leq k_{1}<k_{2}<\cdots<k_{m} \leq n\right\}$ and marked with the multi-index $\left(k_{1}, \ldots, k_{m}\right)$ with the complementary multi-index $\left(k_{1}^{\prime}, \ldots, k_{n-m}^{\prime}\right)$, determines the set of free variables $x^{k_{1}^{\prime}}, \ldots, x^{k_{n-m}^{\prime}}$, vanishing at this corner point.

The minimal and maximal elements of the set $\left\{\frac{\Delta_{k_{1} \cdots k_{m}}^{1}}{\Delta_{k_{1} \cdots k_{m}}^{2}} \mid 1 \leq k_{1}<k_{2}<\cdots<k_{m} \leq n\right\}$ are not necessary the minimum and maximum values of the objective function (1) on the restriction set $\left(2^{\prime}\right)-(3)$, since the condition (3) for the variables that are not free, can be violated. The practical procedure for finding an extremum (for example, a minimum) consists of a consequent verification of the set of free variables, corresponding to the appropriate numeric elements $\frac{\Delta_{k_{1} \ldots k_{m}}}{\Delta_{k_{1} \ldots k_{m}}^{2}}$ of the set, starting from the minimal one. The system of equations $\left(2^{\prime}\right)$ is being solved only for these free variables. In contrast to the simplex method, where the sorting comes from within the admissible set of restrictions to decrease the values of the functions, in the proposed method the direction of sorting is performed from outside to inside the admissible set in the direction of increasing the value of the objective function to the point of the admissible minimum, if it exists. It is believed that in the practical problems the set of corner points which are not valid, that is in violation of the inequalities (3), is much less than the set of the admissible corner points. This is the advantage of the proposed method with respect to the simplex method. The disadvantage is the need to precalculate a large number $2 \cdot C_{n}^{m}$ of determinants of dimension $m \times m$.

\section{Examples of Solution of the Linear Programming Problem with the Method of Exterior Forms}

Example 1. Find extrema of the function

provided that

$$
J(x, y, z)=x+y+z
$$

$2 x+z=1$,

$x, y, z \geq 0$. 
Solution. Find the $1 \times 1$-determinants of two $1 \times 3$-matrices

$$
\begin{aligned}
& \left(\begin{array}{llllll}
1 & 1 & 1
\end{array}\right) \quad \text { and } \quad\left(\begin{array}{llll}
2 & 0 & 1
\end{array}\right) \\
& \Delta_{1}^{1}=1, \Delta_{2}^{1}=1, \Delta_{3}^{1}=1, \Delta_{1}^{2}=2, \Delta_{2}^{2}=0, \Delta_{3}^{2}=1 .
\end{aligned}
$$

Make the list of fractions $\left\{\frac{\Delta_{1}^{1}}{\Delta_{1}^{2}}=\frac{1}{2}, \frac{\Delta_{2}^{1}}{\Delta_{2}^{2}}=\frac{1}{0}, \frac{\Delta_{3}^{1}}{\Delta_{3}^{2}}=1\right\}$.

Put the list in the ascending order $\left\{\frac{\Delta_{1}^{1}}{\Delta_{1}^{2}}=\frac{1}{2}, \frac{\Delta_{3}^{1}}{\Delta_{3}^{2}}=1, \frac{\Delta_{2}^{1}}{\Delta_{2}^{2}}=\frac{1}{0}\right\}$.

All the values of function $J$ at the corner points belong to this list. The free variables of the value $\frac{1}{2}$ are $y$ and $z$. The equations $y=0, z=0$ imply $x=\frac{1}{2} \geq 0$. Therefore, the corner point $(x, y, z)=\left(\frac{1}{2}, 0,0\right)$ is acceptable. This is the minimum point of the function $J$, because the number $\frac{1}{2}$ is the minimal element of the constructed set. The function has no maximum, since there is an element $\frac{1}{0}$ in the list.

Example 2. Find the minimum of the function

$$
J\left(x_{1}, x_{2}, x_{3}, x_{4}, x_{5}\right)=7 x_{3}-x_{4}-3 x_{5}
$$

provided that

$$
\begin{gathered}
\frac{1}{8} x_{1}+\frac{1}{8} x_{3}+\frac{3}{4} x_{4}+\frac{1}{4} x_{5}=1, \\
x_{1}-\frac{8}{9} x_{2}+\frac{1}{9} x_{3}+6 x_{4}-\frac{2}{3} x_{5}=0, \\
x_{1}, x_{2}, x_{3}, x_{4}, x_{5} \geq 0 .
\end{gathered}
$$

Solution. Find the $2 \times 2$-determinants of two $2 \times 5$-matrices

$$
\begin{gathered}
\left(\begin{array}{ccccc}
0 & 0 & 7 & -1 & -3 \\
1 & -\frac{8}{9} & \frac{1}{9} & 6 & -\frac{2}{3}
\end{array}\right) \quad \text { and } \quad\left(\begin{array}{ccccc}
\frac{1}{8} & 0 & \frac{1}{8} & \frac{3}{4} & \frac{1}{4} \\
1 & -\frac{8}{9} & \frac{1}{9} & 6 & -\frac{2}{3}
\end{array}\right) \\
\Delta_{12}^{1}=0, \Delta_{13}^{1}=-7, \Delta_{14}^{1}=1, \Delta_{15}^{1}=3, \Delta_{23}^{1}=\frac{56}{9}, \Delta_{24}^{1}=-\frac{8}{9}, \Delta_{25}^{1}=-\frac{8}{3}, \\
\Delta_{34}^{1}=42 \frac{1}{9}, \Delta_{35}^{1}=-\frac{13}{3}, \Delta_{45}^{1}=18 \frac{2}{3}, \\
\Delta_{12}^{2}=-\frac{1}{9}, \Delta_{13}^{2}=-\frac{1}{9}, \Delta_{14}^{2}=0, \Delta_{15}^{2}=-\frac{1}{3}, \Delta_{23}^{2}=\frac{1}{9}, \Delta_{24}^{2}=\frac{2}{3}, \Delta_{25}^{2}=\frac{2}{9}, \\
\Delta_{34}^{2}=\frac{2}{3}, \Delta_{35}^{2}=-\frac{1}{9}, \Delta_{45}^{2}=-2 .
\end{gathered}
$$


Make the list of fractions

$$
\begin{gathered}
\left\{\frac{\Delta_{12}^{1}}{\Delta_{12}^{2}}=0, \frac{\Delta_{13}^{1}}{\Delta_{13}^{2}}=63, \frac{\Delta_{14}^{1}}{\Delta_{14}^{2}}=\frac{1}{0}, \frac{\Delta_{15}^{1}}{\Delta_{15}^{2}}=-9, \frac{\Delta_{23}^{1}}{\Delta_{23}^{2}}=56, \frac{\Delta_{24}^{1}}{\Delta_{24}^{2}}=-\frac{4}{3}, \frac{\Delta_{25}^{1}}{\Delta_{25}^{2}}=-12,\right. \\
\left.\frac{\Delta_{34}^{1}}{\Delta_{34}^{2}}=63 \frac{1}{6}, \frac{\Delta_{35}^{1}}{\Delta_{35}^{2}}=39, \frac{\Delta_{45}^{1}}{\Delta_{45}^{2}}=-9 \frac{1}{3}\right\} .
\end{gathered}
$$

Put the list in the ascending order

$$
\begin{gathered}
\left\{\frac{\Delta_{25}^{1}}{\Delta_{25}^{2}}=-12, \frac{\Delta_{45}^{1}}{\Delta_{45}^{2}}=-9 \frac{1}{3}, \frac{\Delta_{15}^{1}}{\Delta_{15}^{2}}=-9, \frac{\Delta_{24}^{1}}{\Delta_{24}^{2}}=-\frac{4}{3}, \frac{\Delta_{12}^{1}}{\Delta_{12}^{2}}=0, \frac{\Delta_{35}^{1}}{\Delta_{35}^{2}}=39,\right. \\
\left.\frac{\Delta_{23}^{1}}{\Delta_{23}^{2}}=56, \frac{\Delta_{13}^{1}}{\Delta_{13}^{2}}=63, \frac{\Delta_{34}^{1}}{\Delta_{34}^{2}}=63 \frac{1}{6}, \frac{\Delta_{14}^{1}}{\Delta_{14}^{2}}=\frac{1}{0}\right\} .
\end{gathered}
$$

Check whether the minimal element of the list $\frac{\Delta_{25}^{1}}{\Delta_{25}^{2}}=-12$ is admissible. The free variables corresponding to this element are $x_{1}, x_{3}, x_{4}$. From the equations of the problem, the values of the missing variables are $x_{2}=-3, x_{5}=4$. The point $\left(x_{1}, x_{2}, x_{3}, x_{4}, x_{5}\right)=(0,-3,0,0,4)$ has a negative coordinate, so is not valid.

Check the following element in the ascending order $\frac{\Delta_{45}^{1}}{\Delta_{45}^{2}}=-9 \frac{1}{3}$. The free variables for it are $x_{1}, x_{2}, x_{3}$. The vanishing of the free variables $x_{1}=x_{2}=x_{3}=0$ gives the values of the dependent variables $x_{4}=\frac{1}{3}, x_{5}=3$. The point $\left(x_{1}, x_{2}, x_{3}, x_{4}, x_{5}\right)=\left(0,0,0, \frac{1}{3}, 3\right)$ is admissible. Therefore, it is a point of global minimum of the function $J$ under the given constraints.

\section{Conclusion.}

The paper presents a new simple method, taken from geometry [4,5], for solving the linear programming problem using only the idea of linear dependency of vectors. The method is easily programmable and, in some cases, can compete with the G. B. Danzig simplex method, widely used to solve practical problems [1,2,3].

At the first stage the algorithm calculates the spectrum of all possible values of the objective function at the vertices of the simplex, but without calculating the vertices themselves, using explicite formulas via determinants. The duration of this phase is caused by the need to calculate a large number $2 \cdot C_{n}^{m}$ of $m \times m$-determinants. The second stage deals with sorting the points outside the simplex to the first extreme point belonging to the simplex. In practice, the second step is much shorter than by G. B. Danzig algorithm [1].

\section{References}

[1] G. B. Dantzig, Linear Programming and Extensions, Princeton University Press, 1963.

[2] I. Maros, Computational Techniques of the Simplex Method, Springer Science+Business Media, New York, 2003.

[3] B. A. Murtagh, Advanced Linear Programming: Computation and Practice, McGraw-Hill International Book Company, 1981.

[4] H. Grassmann, Die Ausdehnungslehre. Vollständig und in strenger Form begründet., Berlin: Enslin, 1862.

[5] E. Cartan, Les systèmes différentiels extérieurs et leurs applications géométriques, Hermann, 1945. 